\title{
Behavioral, but not reward, risk modulates activation of prefrontal, parietal, and insular cortices
}

\author{
SCOTT A. HUETTEL \\ Duke University Medical Center, Durham, North Carolina
}

\begin{abstract}
Risky decisions may involve uncertainty about possible outcomes (i.e., reward risk) or uncertainty about which action should be taken (i.e., behavioral risk). Determining whether different forms of risk have distinct neural correlates is a central goal of neuroeconomic research. In two functional magnetic resonance imaging experiments, subjects viewed shapes that had well-learned response-reward contingencies. Magnitude of a monetary reward was held constant within one experiment, whereas expected value was held constant within the other. Response selection, in the absence of behavioral risk, evoked activation within a broad set of brain regions, as had been found in prior studies. However, behavioral risk additionally modulated activation in prefrontal, parietal, and insular regions, within which no effect of reward risk was observed. Reward delivery, in comparison with omission, evoked increased activity in the ventromedial prefrontal cortex and the nucleus accumbens. We conclude that distinct brain systems are recruited for the resolution of different forms of risk.
\end{abstract}

Many decisions are fraught with risk. Doctors and patients must choose among potential courses of action by weighing uncertain benefits and costs, and the manner in which risk is presented guides medical decision making (McNeil, Pauker, Sox, \& Tversky, 1982). When gamblers bet on dice throws, poker hands, or sporting events, they judge the relative probabilities of a set of risky outcomes (Bernoulli, 1738). Both the buying and the selling of insurance depend upon calculated actuarial information and subjective assessments of unlikely but catastrophic events (Johnson, Hershey, Meszaros, \& Kunreuther, 1993). In all of these cases, decisions must be made despite limited knowledge about the likely consequences of chosen actions.

Although the lay usage of risk reflects a single cognitive state of ambiguity or doubt, more precise conceptualizations may be possible. A seminal monograph by F. H. Knight (1921) defined risky decisions as those in which the decider has a priori or statistical knowledge about the probability of likely outcomes. (Note that such situations are fundamentally different from uncertain decisions, in the Knightian sense-i.e., those for which a probability distribution of likely outcomes is not known.) A central consideration of economic theories of decision making has been whether all forms of risk or uncertainty reflect

This study was supported by NIMH Grants MH-70685 and NS-41328. The author thanks E. Gordon, B. Warner, J. Smith, E. Dagenbach, E. Dameron, and R. Sheu for assistance in data collection and analysis and F. Dolcos, G. McCarthy, J. Morris, M. Platt, and C. Stowe for manuscript comments. Correspondence regarding this manuscript should be addressed to S. A. Huettel, Brain Imaging and Analysis Center, 163 Bell Research Drive, Box 3918 DUMC, Durham, NC 27710 (e-mail: scott .huettel@duke.edu). a similar underlying subjective assessment of belief (de Finetti, 1937; Ramsey, 1931; Savage, 1954) or whether different decision processes are invoked depending on the source of risk (Ellsberg, 1961; Epstein \& Wang, 1994; F. H. Knight, 1921). The recent growth of the interdiscipline of Neuroeconomics is predicated on the assumption that information about brain mechanisms can inform these sorts of economic questions (Glimcher, 2003).

In this article, we compare decisions made under two forms of risk, which we label reward risk and behavioral risk. Reward risk reflects limited knowledge about which outcome will occur (i.e., it is a continuous quantity that is probabilistically expressed). In contrast, behavioral risk reflects limited knowledge about which potential action is the optimal one to choose (i.e., it is a categorical variable that is present or absent). To use simple gambles for illustration, if one is given the option to bet with even odds on whether a roll of a standard six-sided die will be greater than 1 , one should obviously take the bet. This gamble incurs reward risk (because one does not know what outcome will occur), but not behavioral risk (because one knows what bet to make). In contrast, betting on whether a coin flip will be heads or tails incurs both behavioral and reward risk. Note that although one can have reward risk without behavioral risk, the opposite is not possible: One will be unsure about one's optimal action only when potential outcomes are unknown or incomparable. Although rational decision makers could ignore behavioral risk (e.g., by always betting heads on a series of coin flips), knowledge of past decisions influences choices even when outcomes are completely random (Barraclough, Conroy, \& Lee, 2004; Huettel, Mack, \& McCarthy, 2002; Lockhead, 1992). 
Electrophysiological and neuroimaging studies have provided strong evidence that reward risk influences the neural responses to rewards themselves. Dopaminergic neurons within the ventral tegmental area of the midbrain respond not only to rewards, but also to stimuli that provide information about the delivery of future rewards (Schultz, 1998; Schultz, Dayan, \& Montague, 1997). This finding was extended by Fiorillo and colleagues, who demonstrated that the activity of such neurons scales directly with the probability that a stimulus predicts future rewards (Fiorillo, Tobler, \& Schultz, 2003). (A second, intriguing result from the same study was the demonstration that the activity of some neurons is greatest for stimuli with maximal reward risk.) Similar results have been reported, in human neuroimaging studies, within regions that receive significant dopaminergic projections from the midbrain, including the nucleus accumbens in the striatum and the ventral prefrontal cortex (Breiter, Aharon, Kahneman, Dale, \& Shizgal, 2001; Knutson, Adams, Fong, \& Hommer, 2001; Knutson, Fong, Bennett, Adams, \& Hommer, 2003). For example, after subjects made a decision that had different levels of reward risk (but no behavioral risk), delay period activity within the anterior cingulate and the ventral prefrontal cortices increased with increased risk (Critchley, Mathias, \& Dolan, 2001).

Less well understood are the effects of risk, whether reward or behavioral, upon brain systems that support decision making. Across a wide range of experimental tasks, decision making in the absence of risk evokes activity within the dorsolateral prefrontal (dlPFC) and parietal cortices (Cohen et al., 1997; Courtney, Ungerleider, Keil, \& Haxby, 1997; McCarthy, Luby, Gore, \& Goldman-Rakic, 1997). The introduction of risk, however, may recruit additional regions of activity, including the frontomedian cortex (FMC), which has been implicated in hypothesis testing and updating of behavior (Goel \& Dolan, 2000; Paulus, Hozack, Frank, \& Brown, 2002; Ullsperger \& von Cramon, 2001; Volz, Schubotz, \& von Cramon, 2003, 2004). Given these demonstrations of generalized effects of risk on brain systems for reward evaluation and decision making, it is critical to investigate the contributions of different forms of risk to decision making.

Our task required subjects to learn stimulus-response contingencies for five possible stimuli and two possible responses (Figures 1A and 1B). Some stimuli were associated with a known response and a guaranteed reward (certainty condition); others were associated with a known response but a probabilistic reward (reward risk condition), and the last could be rewarded for either response (behavioral risk condition). We conducted two experiments in order to control for different potential confounding factors. In Experiment 1, we kept reward magnitude constant and allowed expected value to vary across the stimuli, and in Experiment 2, we kept expected value constant and allowed reward magnitude to vary. The reward risk and behavioral risk conditions were also probabilistically matched in Experiment 2, so that any difference in

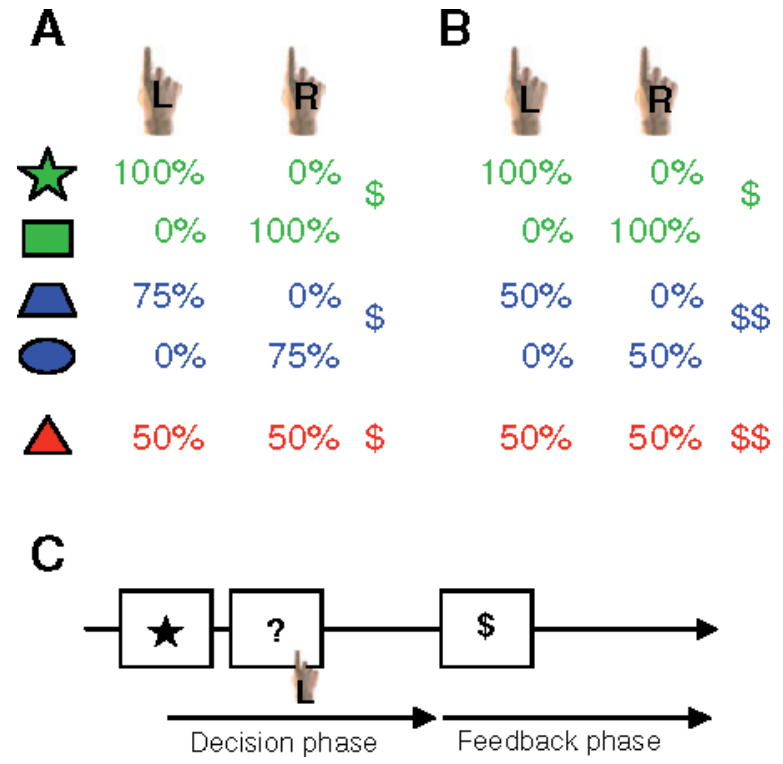

Figure 1. Design of the two experiments. Subjects learned the behavioral and reward contingencies associated with five shapes, each with a different reinforcement probability for left and right buttonpresses. Two of the shapes had a known response and a guaranteed reward (certainty condition; shown in green in all graphs). Two shapes had a known response but an uncertain reward (reward risk condition; blue). And one shape was rewarded for each response with equal frequency (behavioral risk condition; red). The shape colors are shown here for clarity; during the experiment, all the shapes were presented in the same color. (A) In Experiment 1, reward magnitude was held constant across the three experimental conditions $(\mathbf{\$ 0 . 2 5})$, while expected value differed. (B) In Experiment 2, expected value was held constant across the conditions, while reward magnitude differed $\mathbf{( \$ 0 . 1 5}$ or \$0.30). (C) At the beginning of each trial, a single shape was presented. Following a short, fixed delay, a question mark cued the subject to indicate his or her response with a left or right buttonpress on a response box. Following a longer, variable delay, the subject received feedback indicating whether (\$ and \$\$) or not $(X)$ he or she received a monetary reward.

activation between them could be attributed to the effects of behavioral risk.

By jittering the interval between the decision and the reward phases of this task (Figure 1C), we could examine the effects of risk on each phase independently, using functional magnetic resonance imaging (fMRI). We hypothesized that there would be increased activation to the behavioral risk condition, in comparison with the reward risk condition, within the prefrontal, parietal, and insular cortices. That is, within these regions, which have previously been implicated in executive control, risk would matter only when it influenced behavioral selection. Alternatively, we could observe increased activation in these regions to any form of risk, regardless of its source. We further expected that reward delivery would modulate activation in ventral brain regions that are targeted by dopaminergic reward-related inputs, including the nucleus accumbens and the ventromedial prefrontal cortex. 


\section{GENERAL METHOD}

\section{Subjects}

In Experiment 1, data from 12 healthy young adult volunteers (age range, 18-29 years; 6 of them female and 6 male) were included in the analyses. Experiment 2 comprised data from 11 healthy volunteers (age range, 18-34 years; 9 of them female and 2 male). All had accurate behavioral performance and less than 1 voxel maximum head motion, and all gave written informed consent. Across both experiments, an additional 6 subjects completed data collection but were excluded from statistical analysis, due to unacceptable head motion or behavioral noncompliance. This study was approved by the Institutional Review Board of Duke University Medical Center.

\section{Experimental Design}

On each trial, the subjects viewed one of five shapes, made a buttonpress response, and viewed a feedback stimulus that signified whether or not they received a reward. Each shape was associated with a different set of response-reward contingencies (see Figures 1A and 1B). Two were associated with a guaranteed reward for a correct button response (e.g., a star or a rectangle). These two shapes composed the certainty condition. Another two were associated with a probabilistic reward for pressing the correct button (e.g., a trapezoid or an oval). These two shapes composed the reward risk condition. For each of the above stimuli, an incorrect response was never rewarded. A fifth stimulus (e.g., a triangle) was associated with a $50 \%$ chance of reward for either of the two buttonpresses. This shape composed the behavioral risk condition. The subjects were instructed that the rewarded stimulus in this condition would vary randomly from trial to trial. We expected that the subjects would nevertheless use irrelevant patterns in the reward sequence to control their behavior (Huettel et al., 2002), so that regions associated with the generation of behavior would be differentially active on those trials, in comparison with the other conditions.

Across the three conditions, reward probabilities were $100 \%$, $75 \%$, and $50 \%$ and $100 \%, 50 \%$, and 50\% in Experiments 1 and 2, respectively. To minimize practice or learning effects during the fMRI session, all the subjects were explicitly told these stimulus-response contingencies, and all participated in preliminary practice sessions in a behavioral testing laboratory. The subjects were not paid for their practice session choices.

Stimulus timing was similar in both experiments (Figure 1C). At the outset of each trial, the decision shape was presented for $250 \mathrm{msec}$. Then, following a fixed 3,000-msec onset-to-onset delay, a response cue (?) was presented for $1,000 \mathrm{msec}$. The subjects then pressed one of two response box buttons with their right hand, and only responses during that interval were counted for subsequent reward. Then, after a variable 2,000- to 8,000 -msec delay (onset to onset), a 1,000-msec feedback stimulus indicated the receipt of a reward (\$ or \$\$) or not (X). The next trial began 2,000-8,000 msec after the offset of the feedback stimulus. In Experiment 1, all correct responses were rewarded at the same rate, with each dollar sign corresponding to $\$ 0.25$. In Experiment 2, responses in the certainty condition were rewarded with one dollar sign, or $\$ 0.15$, whereas responses in the other two conditions were rewarded with two dollar signs, or $\$ 0.30$. Thus, reward size was held constant in Experiment 1, and stimulus expected value was held constant in Experiment 2.

All the subjects participated in six experimental runs, each approximately $10 \mathrm{~min}$ in length, for a total of about 250 trials. The task was presented using the Psychophysics Toolbox (Brainard, 1997; Pelli, 1997) for MATLAB (Mathworks, Inc.) and the JoyStk ActiveX control (Mabry Software).

\section{fMRI Data Acquisition}

Identical data acquisition methods were used for the two experiments. Images were acquired using a 4.0-T General Electric (Waukesha, WI) MRI scanner. We collected whole-brain blood-oxygenation-leveldependent (BOLD) images, using a high-throughput $\mathrm{T}_{2} *$-weighted spiral-out pulse sequence (TR, 1,500 msec; TE, $35 \mathrm{msec}$; flip angle, $\left.60^{\circ}\right)$. We collected data from 34 axial slices with near-isotropic voxels of $3.75 * 3.75 * 3.8 \mathrm{~mm}$. High-resolution 3-D full-brain SPGR images were acquired to aid in normalization.

\section{fMRI Data Analysis}

Identical data analysis methods were used for the two experiments. Functional images were corrected for subject motion and time of acquisition within a TR and were normalized into a standard stereotaxic space (Montreal Neurological Institute, MNI) for intersubject comparison, using SPM99 (Wellcome Department of Cognitive Neurology, University College London, 1999). A smoothing filter of 6-mm width was applied following normalization. We excluded individual trials from subsequent analysis on the basis of three quality assurance measures: transient movement greater than $1 \mathrm{~mm}$ during the trial epoch, a change in average signal intensity across the brain greater than \pm 2 standard deviations, or a change in mean signal greater than \pm 2 standard deviations between any consecutive time points

From the overall time series, we extracted epochs that were timelocked to the onset of the decision stimulus (decision phase) and to the onset of the reward stimulus (feedback phase). We note that we do not directly contrast decision and reward phase activity, for two reasons: First, the phases necessarily differed in their stimulus and response requirements, and second, our primary interest was in the effects of experimental condition within a phase. The extracted epochs consisted of the 19 time points from 5 TRs before the decision or reward stimulus through 13 TRs after. Each epoch was baseline corrected to the mean across time points $0-3 \mathrm{sec}$. All analyses collapse over both experiments, but we demonstrate the common activation patterns in the critical behavioral risk comparisons by interrogating the active regions of interest (ROIs) in each experiment independently (Figures 3-5).

To identify brain regions that support response selection in the absence of behavioral risk, we examined the contrast of the combined certainty and reward risk conditions to baseline, collapsing across the two experiments. To avoid making assumptions about the shape of the fMRI hemodynamic response, we identified voxels whose maximal BOLD response at the peak of the hemodynamic response (4-6 TRs following decision stimulus) was significantly different from zero. We calculated significance values for each subject for the comparison at each of the three time points surrounding the peak, and then we subjected those significance values to a second-order random effects analysis across subjects. Given the size of our sample and the large number of trials, we used full Bonferroni correction for $\sim 47,000$ voxels in our normalized brain and three time points of statistical test, along with a minimum cluster size of 6 voxels. In Table 1, we report the foci of activation whose maxima passed a corrected threshold of $p<.001$ ( $t>8.6$; uncorrected $p<7.1 *$ $10^{-9}$ ); region extent (see also Figures 2 and 5) shows all voxels passing a corrected threshold of $p<.05(t>6.8$; uncorrected $p<3.5 *$ $10^{-7}$ ). The use of such conservative threshold values, given the large amount of data included in the analysis, provides very high confidence that activation in the regions identified is associated with the decision phase of the task when no behavioral risk is present.

We then identified voxels whose activation differed between trials involving no behavioral risk (certainty and reward risk) and trials involving behavioral risk. Procedures were similar to that described above for response selection, but we used a lower threshold, since we were investigating differences between conditions. The same procedures and threshold values were used for the comparison of rewarded and unrewarded trials, for which epochs were time-locked to reward delivery. All the regions reported in Tables 2 and 3 had a maximal significance value that passed a threshold of $p<.0001$ (corrected for three time points of test; $t>4.9$ ), with six or more contiguous voxels passing a threshold of $p<.001$ (as plotted in Figures 3-5).

All plotted hemodynamic waveforms show change in BOLD signal over a 13.5-sec period (9TRs) following the onset of the decision 
Table 1

Regions Exhibiting Decision Phase Activity to Response Selection in the Absence of Behavioral Risk

\begin{tabular}{lcrrrrr}
\hline \multicolumn{1}{c}{ Region } & & \multicolumn{2}{c}{ MNI Centroid } & & \\
\cline { 2 - 4 } \multicolumn{1}{c}{ Laterality } & \multicolumn{1}{c}{$x$} & \multicolumn{1}{c}{$y$} & \multicolumn{1}{c}{$z$} & Volume (cc) & Max $t$ \\
\hline Middle frontal gyrus & $\mathrm{L}$ & -42 & 46 & 25 & 0.6 & 8.6 \\
Putamen & $\mathrm{L}$ & -25 & 11 & 4 & 4.3 & 9.7 \\
Frontomedian cortex & $\mathrm{B}$ & 0 & 7 & 46 & 21.8 & 12.5 \\
Putamen & $\mathrm{R}$ & 25 & 7 & 7 & 5.6 & 8.8 \\
Precentral gyrus & $\mathrm{R}$ & 53 & 4 & 7 & 1.8 & 8.7 \\
Insula & $\mathrm{L}$ & -49 & 0 & 7 & 4.5 & 10.0 \\
Putamen & $\mathrm{L}$ & -21 & 0 & 11 & 2.1 & 9.4 \\
Inferior frontal sulcus & $\mathrm{L}$ & -60 & 0 & 39 & 0.8 & 9.4 \\
Inferior frontal sulcus & $\mathrm{L}$ & -46 & -4 & 35 & 1.1 & 9.0 \\
Insula/putamen & $\mathrm{L}$ & -28 & -11 & 7 & 6.3 & 9.7 \\
Precentral gyrus & $\mathrm{L}$ & -35 & -21 & 60 & 9.8 & 11.0 \\
Postcentral gyrus & $\mathrm{L}$ & -53 & -28 & 21 & 1.0 & 8.6 \\
Postcentral gyrus & $\mathrm{L}$ & -53 & -28 & 53 & 2.2 & 9.3 \\
Postcentral gyrus & $\mathrm{L}$ & -39 & -39 & 53 & 18.1 & 10.9 \\
Inferior temporal gyrus & $\mathrm{L}$ & -56 & -42 & -21 & 0.8 & 8.7 \\
Fusiform/cerebellum & $\mathrm{R}$ & 35 & -63 & -21 & 21.1 & 11.2 \\
Superior parietal lobule & $\mathrm{L}$ & -25 & -67 & 53 & 9.3 & 11.2 \\
Fusiform/cerebellum & $\mathrm{L}$ & -32 & -67 & -18 & 19.2 & 10.3 \\
Superior parietal lobule & $\mathrm{R}$ & 7 & -70 & 53 & 4.3 & 10.3 \\
Cerebellum & $\mathrm{B}$ & 0 & -77 & -21 & 3.0 & 10.5 \\
\hline
\end{tabular}

stimulus (Figures 3-5) or the reward stimulus (Figure 6). On each figure, scaling of the $y$-axis is indicated by values on the plot at the upper left, and common scales were used for all the graphs within a figure. The waveforms were calculated by identifying regions of contiguous suprathreshold voxels around the centroids of significant activity indicated above each plot and then calculating the percentage of signal change over time across all voxels in the ROI. All rendered brains were created using MRIcro (Chris Rorden, University of South Carolina), with the locations of any cutting plane indicated in MNI coordinates.

\section{RESULTS}

\section{Behavioral Data}

In neither experiment were the subjects' response times or response accuracy influenced by risk, ruling out task difficulty as an explanation for subsequent fMRI results.
In Experiment 1, mean response times across subjects to certainty, reward risk, and behavioral risk trials were 492, 494, and $487 \mathrm{msec}$, respectively. In Experiment 2, mean response times across subjects to certainty, reward risk, and behavioral risk trials were 452, 451, and $437 \mathrm{msec}$, respectively. No response time differences between conditions within an experiment were significant (all $p \mathbf{s}>.1$ ).

We defined correct responses as those for which the subject selected a stimulus that could be rewarded (i.e., either response would count for behavioral risk trials) within the 1,000-msec response window. Accuracy across all the conditions in both experiments ranged from $93.4 \%$ to $95.5 \%$, and no two conditions significantly differed from each other (all $p \mathrm{~s}>.1$ ).

Most, but not all, of the subjects allocated their responses in the behavioral risk condition relatively equally

Table 2

Regions Whose Decision Phase Activity Was Modulated by Behavioral Risk

\begin{tabular}{lcrrrrr}
\hline \multicolumn{1}{c}{ Region } & & \multicolumn{3}{c}{ MNI Centroid } & & \\
\cline { 2 - 4 } \multicolumn{1}{c}{ Laterality } & \multicolumn{1}{c}{$x$} & \multicolumn{1}{c}{$y$} & \multirow{2}{c}{$z$} & Volume (cc) & Max $t$ \\
\hline Frontopolar cortex & $\mathrm{R}$ & 28 & 60 & 7 & 6.1 & 6.9 \\
Frontopolar cortex & $\mathrm{L}$ & -39 & 53 & 4 & 7.3 & 6.0 \\
Frontomedian cortex & $\mathrm{B}$ & -4 & 28 & 39 & 17.8 & 7.6 \\
Middle frontal gyrus & $\mathrm{R}$ & 42 & 28 & 39 & 3.6 & 6.1 \\
Insula & $\mathrm{R}$ & 39 & 21 & 0 & 4.6 & 6.8 \\
Insula & $\mathrm{L}$ & -35 & 18 & 0 & 5.1 & 6.8 \\
Superior frontal gyrus & $\mathrm{R}$ & 32 & 14 & 60 & 8.8 & 7.0 \\
Superior frontal gyrus & $\mathrm{L}$ & -21 & 14 & 63 & 0.7 & 5.5 \\
Putamen & $\mathrm{R}$ & 18 & 0 & 4 & 1.0 & 7.6 \\
Thalamus & $\mathrm{R}$ & 14 & -25 & 18 & 0.7 & 5.5 \\
Posterior cingulate gyrus & $\mathrm{B}$ & 0 & -28 & 32 & 3.7 & 5.3 \\
Supramarginal gyrus & $\mathrm{R}$ & 49 & -56 & 46 & 7.0 & 5.9 \\
Supramarginal gyrus & $\mathrm{L}$ & -35 & -63 & 46 & 4.7 & 5.6 \\
Cerebellum & $\mathrm{L}$ & -35 & -70 & -32 & 1.1 & 6.2 \\
Cerebellum & $\mathrm{R}$ & 11 & -77 & 49 & 2.3 & 6.1 \\
\hline
\end{tabular}


between the two responses. Across the entire sample of 23 subjects, the mean proportion of left-button responses was .43. Two subjects responded exclusively, or nearly so, with one of the buttons. Examination of the mean activation data excluding these 2 subjects did not suggest that their inclusion or exclusion had a meaningful effect on the results.

\section{Response Selection Effects, Independent of Behavioral Risk}

We initially identified brain regions with significant activation even when behavioral risk was not present by combining across the certainty and reward risk conditions (see Figure 2 and Table 1). Significant decision phase activity was observed in the parietal cortex, the dlPFC, the medial frontal lobe, the basal ganglia, the thalamus, and the insula. Additional foci of activity were observed in motor/sensory areas around the left central sulcus and in the posterior and ventral visual cortices. Activation was similar across the two experiments, and so Figure 2 collapses over them.

\section{Behavioral Risk Effects}

We next evaluated the effects of behavioral risk, independent of any response selection effects, by contrasting decision phase activity in the behavioral risk condition to decision phase activity across the other two conditions (which had no behavioral risk). Regions exhibiting significant behavioral risk effects are indicated in Table 2 . Note that the inclusion of the 50\%-rewarded reward risk condition in Experiment 2 allows these effects of behavioral risk to be attributed to decision processes and not to reward probability or expected value.

Within frontal regions (Figure 3A), we found that behavioral risk evoked increased activation in the frontopolar cortex, the insular cortex, and the superior and middle frontal gyri. Within all regions, activation patterns were similar between the two experiments (Figures 3B and $3 \mathrm{C}$ ), indicating that differences in reward amplitude or expected value did not cause the behavioral risk effect. Furthermore, activation was absent or greatly reduced when behavioral risk was absent. The observed insular ROI was anterior to that found for response selection, and within it we found only activation associated with the behavioral risk condition.

Significant effects of behavioral risk were also observed within the parietal cortex, along the supramarginal gyrus bilaterally (Figure 4A). Activation patterns within the region of activation were again similar between the two experiments (Figures 4B and 4C). Within the left supramarginal gyrus, activation was present in all three conditions, with the greatest amplitude observed in the
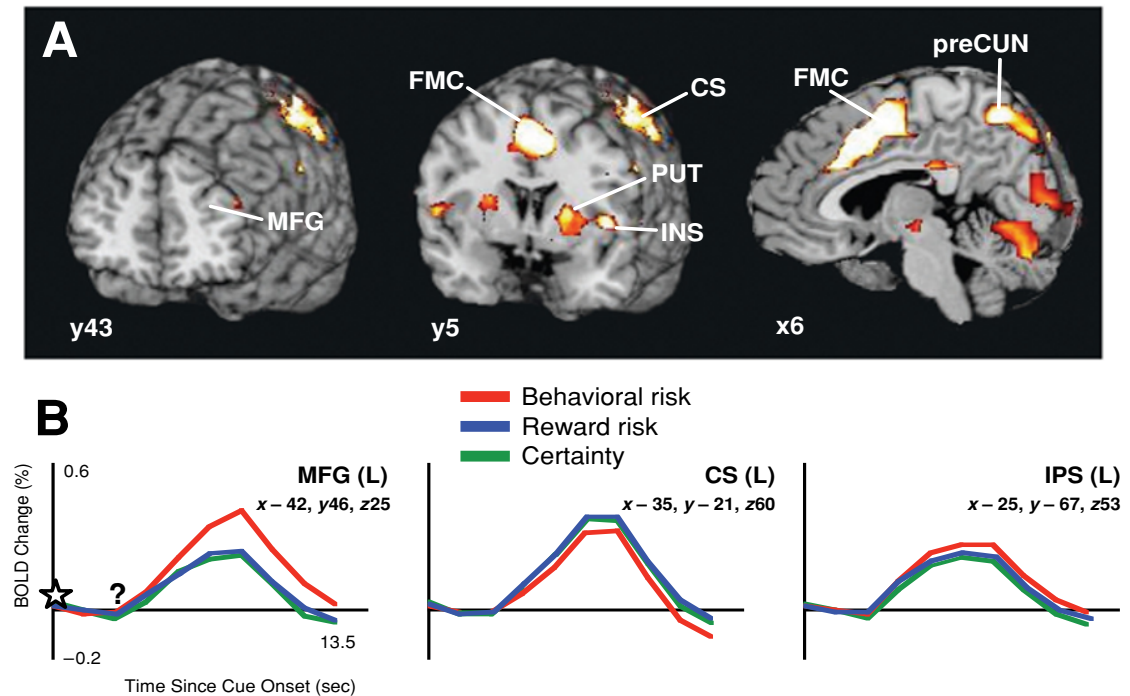

Figure 2. Regions exhibiting significant decision phase activity related to response selection in the absence of behavioral risk (i.e., combining across the certainty and reward risk conditions). (A) Visible in these cross-sectional views are significant foci of activity in the anterior middle frontal gyrus (MFG), the frontomedial cortex (FMC), the insula (INS), the putamen (PUT), the central sulcus (CS), and the precuneus (preCUN). Color maps indicate Bonferonni-corrected significance of $p<.05$ (red; uncorrected $p<3.5 * 10^{-7}$ ) to greater than $p<.0001$ (yellow; uncorrected $p<7.1 * 10^{-10}$ ). (B) Within these regions, the fMRI hemodynamic response peaked approximately $4.5-6 \mathrm{sec}$ following the response cue, and activation amplitude did not significantly differ across experimental conditions. In this and all the subsequent figures, the $x$-axis indicates a 13.5-sec epoch following the presentation of the decision stimulus (indicated by a star). The response cue was presented 3 sec later. The scale of the $y$-axis (percentage of BOLD signal change) is indicated by endpoint labels and is kept constant across all the charts within a figure. Green indicates certainty, blue indicates reward risk, and red indicates behavioral risk. Centroids of the regions of interest and slice locations are provided in MNI coordinates. 


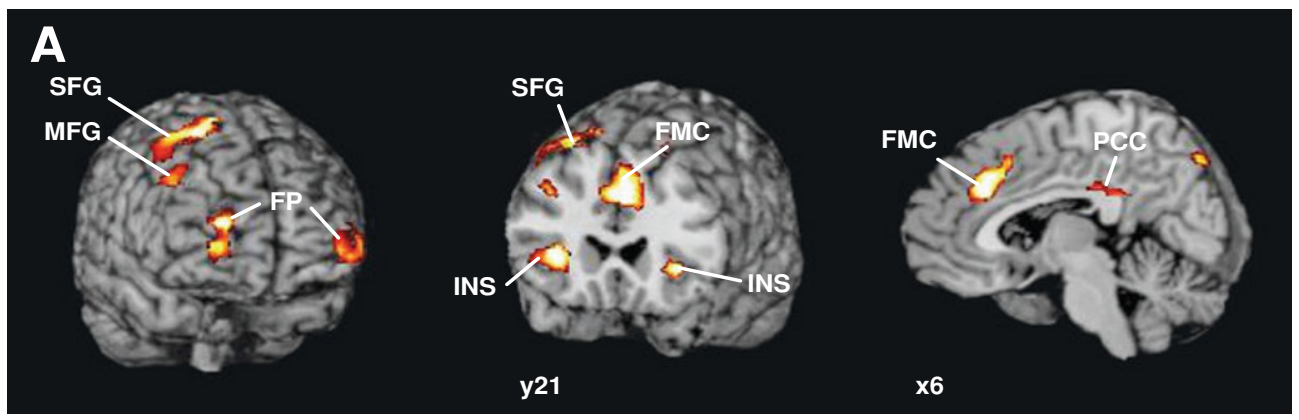

\section{B Constant Reward}
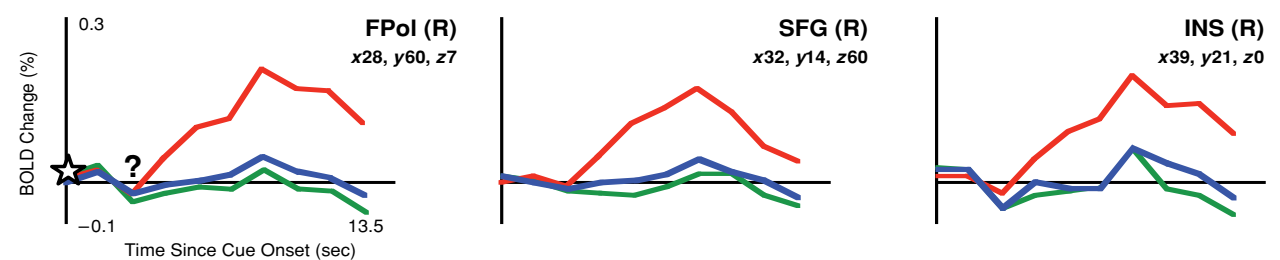

\section{Constant Expected Value}
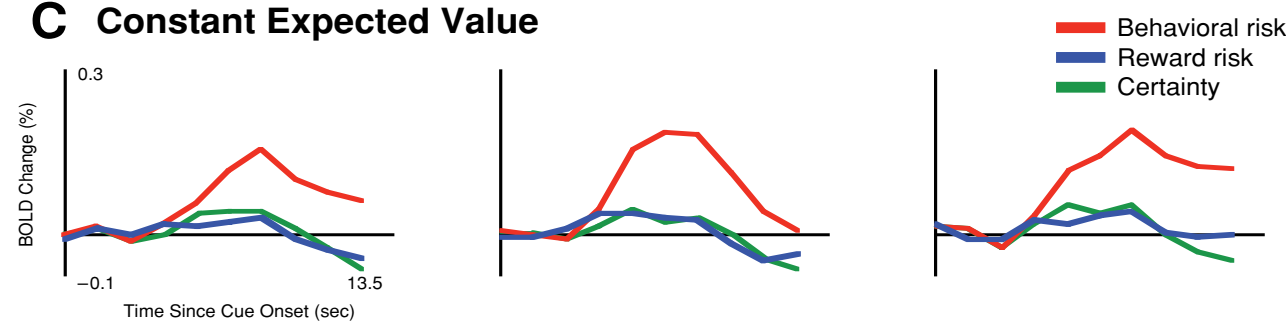

Figure 3. Frontal and midline regions exhibiting significant effects of behavioral risk. (A) Significantly greater activation was observed in the behavioral risk condition than in the other two conditions within the frontopolar cortex (FP), the middle frontal gyrus (MFG), the posterior superior frontal gyrus (SFG), the frontomedian cortex (FMC), and the posterior cingulate cortex (PCC). Color maps on this and the subsequent figures indicate significance values from at least $p<.001$ (red) to greater than $p<.00001$ (yellow). (B) In Experiment 1, which kept reward constant across the experimental conditions, activation within these regions was largely restricted to the behavioral risk condition, as shown on the plotted hemodynamic time courses. (C) Similar behavioral risk effects were found in Experiment 2, even though that experiment used a different subject sample and a different set of stimulus-reward contingencies.

behavioral risk condition. But within the right supramarginal gyrus, significant activation was present only for behavioral risk.

Activation in the medial frontal lobe evinced a gradient across space. Shown in Figure 5A is a mid-sagittal slice, upon which is overlaid the significance maps for both the main effect of the decision phase (red-yellow) and the differential effect of behavioral risk (blue-white). Activation was observed along a posterior-to-anterior axis corresponding roughly to Brodmann areas (BAs) 6 and 8 and extending into posterior BA 9. Plotted in Figures 5B and $5 \mathrm{C}$ are mean hemodynamic time courses for Experiments 1 and 2, respectively, at five equally spaced voxels along that axis. In the posterior parts of the medial frontal lobe, in BA 6, activation was identical across the experimental conditions, likely reflecting the similar motor demands. But when moving forward into BA 8 , activation was present in all three conditions, but greatest in the behavioral risk condition. In the most anterior regions exhib- iting a response, in posterior BA 9, activation was present only in the behavioral risk condition. Activation patterns were again similar between the two experiments, despite their different response-reward contingencies.

We additionally compared the certain and reward risk conditions, but no region passed significance testing similar to that for the behavioral risk effect. Thus, we found no evidence for selective effects of reward risk, at least for decisions with well-learned stimulus-response contingencies.

\section{Reward Effects}

Although not primary to the goals of the study, we also investigated changes in brain activation associated with the delivery or omission of reward signals. Presented in Table 3 are foci where significantly greater activation was observed following reward delivery, in comparison with reward omission. In Figure 6, we highlight two regions of significant reward-related activation. Shown in the coronal slice at the left is activation in the left nucleus accum- 


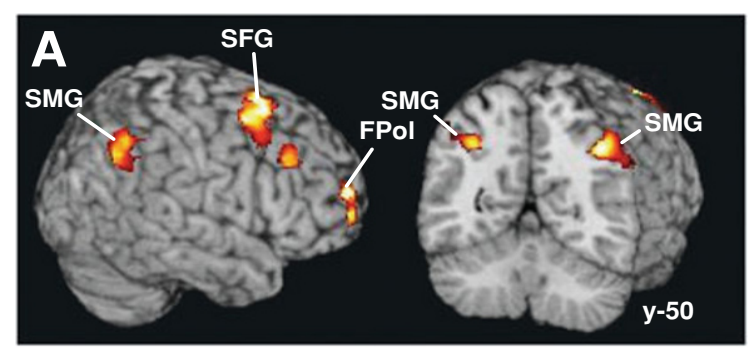

\section{B Constant Reward}

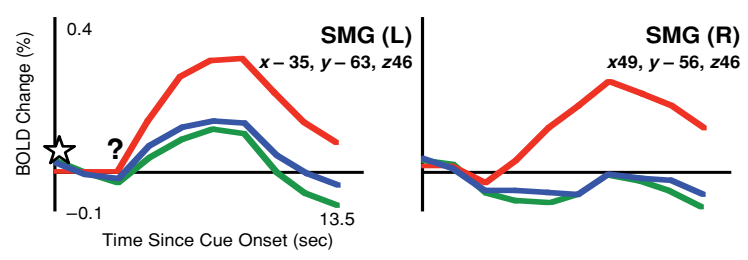

\section{Constant Expected Value}

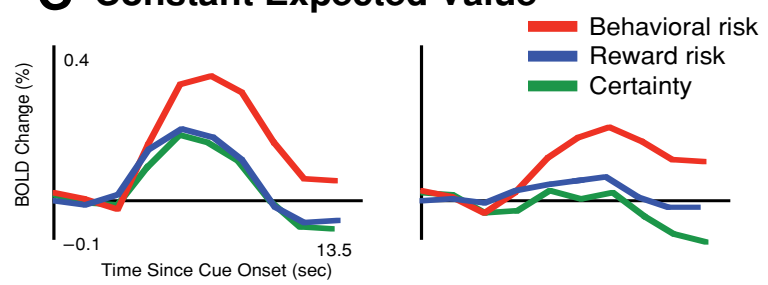

Figure 4. Parietal regions exhibiting significant effects of behavioral risk. (A) A significant increase in activation in the behavioral risk condition was observed in the supramarginal gyrus (SMG) bilaterally. (B) In Experiment 1 (constant reward), examination of the time courses of activation within the SMG regions of interest revealed a much larger response in the behavioral risk condition than in the other two conditions, which did not differ from each other. An increase above baseline in the absence of behavioral risk was found in the left, but not the right, SMG. (C) A similar behavioral risk effect was found in Experiment 2 (constant expected value), replicating the pattern of results from the prior experiment.

bens, which was found for the contrast between rewarded and unrewarded behavioral risk trials. Shown in the sagittal slice at the right is activation in the ventromedial prefrontal cortex, which was found for the contrast between rewarded and unrewarded reward risk trials.

\section{DISCUSSION}

The results provide strong evidence that the presence of behavioral risk significantly increases activation in regions of the lateral prefrontal, medial prefrontal, and lateral parietal cortices. The behavioral risk effect did not result from increased response difficulty, since the response was delayed in time from its cuing stimuli, whereas response time and response accuracy were matched across conditions. Nor was it a consequence of differential reward magnitudes or expected values across stimuli, since reward magnitude was kept constant in Experiment 1 and expected value was kept constant in Experiment 2. And these regions were not differentially activated by differences in outcome risk when behavioral risk was not present.

\section{Response Selection}

The successful matching of a well-practiced behavior to a well-learned stimulus, as in the present certainty and reward risk conditions, is a simple form of response selection. Unlike more complex situations that allow for variability in stimulus-response mappings, as in the present behavioral risk condition, response selection does not require subjects to make decisions between potential responses. For example, many response selection paradigms, such as the common oddball, go/no-go, or twostimulus choice tasks, require subjects to learn a particular stimulus-response mapping that can be readily applied to each stimulus. In these tasks and many others, each stimulus must be identified or classified as it occurs, and then the correct response must be selected for that stimulus or stimulus class. Nevertheless, the simplicity of such tasks belies substantial demands for executive control (Bunge, 2004).

Using a wide range of experimental paradigms, neuroimaging studies have demonstrated that response selection evokes activation in a consistent set of brain regions, including the lateral prefrontal, medial prefrontal, insular, and posterior parietal cortices, as well as the basal ganglia and thalamus. In the oddball paradigm, for example, responses to infrequent target stimuli evoke activation in this system, but equally infrequent novel stimuli with no response requirements do not (Clark, Fannon, Lai, Benson, \& Bauer, 2000; Huettel, Misiurek, Jurkowski, \& McCarthy, 2004; Kirino, Belger, Goldman-Rakic, \& McCarthy, 2000; McCarthy et al., 1997; Stevens, Skudlarski, Gatenby, \& Gore, 2000). Lateral prefrontal activation in response selection tasks has been shown to be independent of response changes themselves (Huettel \& McCarthy, 2004) but dependent on the degree to which a particular stimulus is expected (Huettel et al., 2002). Furthermore, introducing a spatial incompatibility into the response selection process (e.g., respond left when a stimulus is presented on the right) increases activation in these regions (Huettel \& McCarthy, 2004; Jiang \& Kanwisher, 2003a, 2003b; Schumacher, Elston, \& D'Esposito, 2003), consistent with long-standing behavioral data (Simon \& Baker, 1995; Simon \& Small, 1969). All of these paradigms share a common characteristic: Before each stimulus is presented, there are multiple potential responses, which are, in turn, resolved into a single well-practiced response when the stimulus occurs.

The present results are consistent with those in this large prior literature, in that we found that simple response selection with a known reward outcome (i.e., the certainty condition) evoked activation within a similar set of regions. Behavioral risk is thus unnecessary for activation in these regions. However, we also included a condition that involved simple response selection with an unknown outcome, reward risk, and found similar patterns of activation across both experiments. Nor did examination of each 
Table 3

Regions Whose Reward Phase Activity Was Modulated by Reward Delivery or Omission Within the Behavioral Risk and Reward Risk Conditions

\begin{tabular}{|c|c|c|c|c|c|c|}
\hline \multirow[b]{2}{*}{ Region } & \multirow[b]{2}{*}{ Laterality } & \multicolumn{3}{|c|}{ MNI Centroid } & \multirow[b]{2}{*}{ Volume (cc) } & \multirow[b]{2}{*}{$\operatorname{Max} t$} \\
\hline & & $x$ & $y$ & $z$ & & \\
\hline \multicolumn{7}{|c|}{ Behavioral Risk } \\
\hline Lateral orbitofrontal cortex & $\mathrm{L}$ & -43 & 41 & -7 & 1.6 & 5.8 \\
\hline Inferior frontal gyrus & $\mathrm{L}$ & -51 & 22 & -15 & 0.4 & 7.6 \\
\hline Nucleus accumbens & $\mathrm{B}$ & 1 & 7 & -8 & 3.3 & 6.3 \\
\hline Superior temporal gyrus & $\mathrm{R}$ & 58 & 2 & -5 & 2.2 & 7.6 \\
\hline Superior temporal gyrus & $\mathrm{R}$ & 62 & -18 & 11 & 1.8 & 6.0 \\
\hline Superior temporal gyrus & $\mathrm{L}$ & -63 & -30 & -11 & 1.0 & 6.2 \\
\hline Postcentral gyrus & $\mathrm{L}$ & -33 & -38 & 70 & 1.8 & 6.6 \\
\hline Posterior cingulate gyrus & $\mathrm{B}$ & 7 & -43 & 44 & 1.0 & 5.9 \\
\hline Cerebellum & $\mathrm{R}$ & 22 & -61 & -22 & 1.7 & 5.9 \\
\hline Angular gyrus & $\mathrm{L}$ & -48 & -74 & 35 & 0.6 & 6.0 \\
\hline Lateral occipital gyrus & $\mathrm{R}$ & 44 & -83 & -15 & 2.2 & 5.8 \\
\hline Lateral occipital gyrus & $\mathrm{L}$ & -33 & -96 & -14 & 1.8 & 6.2 \\
\hline Lateral occipital gyrus & $\mathrm{R}$ & 11 & -106 & 0 & 1.2 & 5.9 \\
\hline \multicolumn{7}{|c|}{ Reward Risk } \\
\hline Ventromedial prefrontal & & & & & & \\
\hline cortex & B & -6 & 57 & 1 & 10.1 & 7.8 \\
\hline Superior frontal gyrus & $\mathrm{R}$ & 21 & 38 & 49 & 2.6 & 7.0 \\
\hline Superior frontal gyrus & $\mathrm{L}$ & -25 & 28 & 51 & 9.3 & 6.7 \\
\hline Fusiform gyrus & $\mathrm{R}$ & 45 & -41 & -16 & 1.7 & 5.5 \\
\hline Precuneus & $\mathrm{L}$ & -8 & -55 & 30 & 4.2 & 6.4 \\
\hline Lateral occipital gyrus & $\mathrm{R}$ & 36 & -89 & -12 & 6.6 & 6.0 \\
\hline Lateral occipital gyrus & $\mathrm{R}$ & 23 & -101 & -3 & 3.2 & 6.4 \\
\hline
\end{tabular}

experiment individually suggest any effects of reward probability (Experiment 1) or of reward probability and reward magnitude (Experiment 2). Our results suggest that activation of these regions during simple response selection does not depend on risk in their outcome contingencies, at least for the task conditions tested in the present experiments. Such a result does not rule out, however, an influence of reward/affective information under other circumstances (i.e., larger rewards). The lateral prefrontal cortex, in particular, receives substantial inputs from dopaminergic reward systems (Fuster, 1997), and monetary rewards have been shown to influence lateral prefrontal cortex activation in a working memory task (Pochon et al., 2002).

\section{Behavioral Risk}

The behavioral risk condition differs from the others in that it allows subjects to select between two potential responses, each equally likely to be rewarded. Thus, all response strategies are equally valuable, and subjects would receive the same rewards if they always pressed one button (i.e., avoided making any decisions) as if they randomized their responses between the buttons. Nevertheless, in such situations, people tend to match responses to their frequency of reinforcement (Herrnstein, 1961), perhaps attempting to anticipate patterns in the random sequence (Lockhead, 1992). The majority of our subjects did, in fact, switch their response frequently over trials, making it reasonable to discuss the behavioral risk condition as involving decision processes not present in the other two conditions.
Simple decision making has been shown to activate brain regions beyond those required for response selection. Paulus and colleagues used a basic two-choice task in a series of studies to examine the effects of decision making and subject strategy. When comparing a condition involving free decisions with a condition requiring a response based on the location of a visible stimulus, they found that decision making evoked greater activation in the anterior prefrontal cortex, the insular cortex, the inferior parietal lobule, and the precuneus, among other regions (Paulus et al., 2001). These regions are generally similar to those found for behavioral risk in the present study (see Table 2), although we found additional activation in the medial frontal lobe, the basal ganglia, and the posterior superior frontal gyrus. Our results complement and extend the conclusions of Paulus and colleagues, due to our inclusion of differential reward contingencies across stimuli. We show that activation of these regions is not simply a function of uncertainty in the outcome, since both the reward risk and the behavioral risk conditions induce such uncertainty. Within Experiment 2, in particular, these two conditions were equated for reward probability and magnitude, yet there was a dramatic difference in the activation evoked. Thus, the present results indicate that uncertainty in the needed response - not the likely outcome-modulates activation in these regions.

An unexpected and suggestive result was the finding of a gradient in the behavioral risk effect along the medial surface of the frontal lobe (Figure 5). There has been longstanding and significant interest in the role of the anterior cingulate cortex in behavioral control, response monitor- 

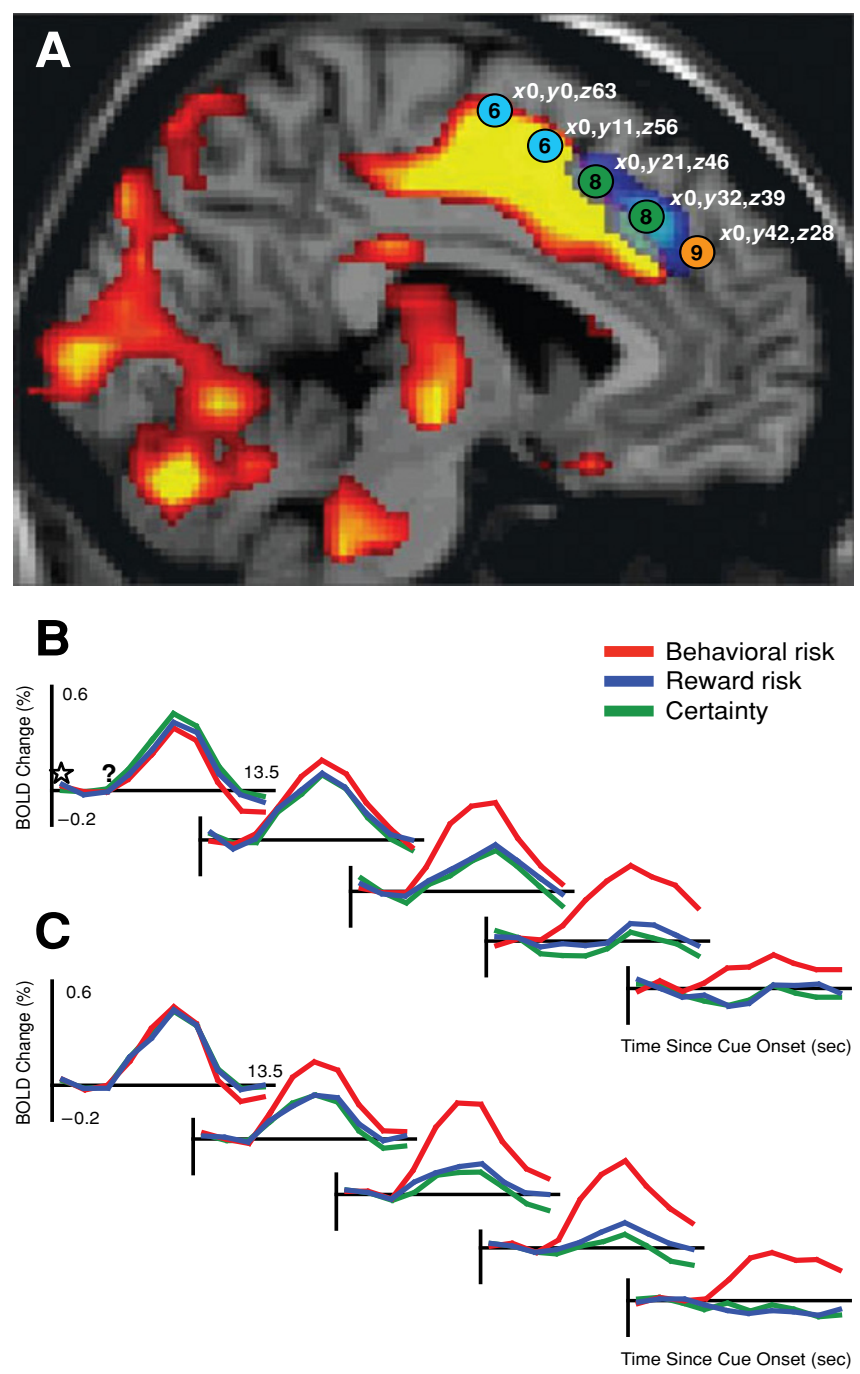

Figure 5. Response selection and behavioral risk effects in the medial frontal lobe. (A) Shown in this sagittal view are activation maps associated with response selection (red-yellow) and behavioral risk (blue-white) effects, presented with similar threshold ranges $(p<0.001$ to $p<.0001)$. To examine changes in the response patterns across the frontomedian cortex, we selected five equally spaced voxels along a posterior-to-anterior axis. Approximate locations and Brodmann areas (BAs) are shown in the colored circles, and exact coordinates are indicated on the figure. (B) Using the data from Experiment 1, we determined the mean time course of activity within each of these voxels for each condition. Visible is a transition from similar responses in all the conditions in the posterior frontomedian cortex (BA 6) to responses only in the behavioral risk condition in more anterior regions (BAs 8 and 9). (C) A very similar pattern of activation was observed in Experiment 2, providing evidence for the reproducibility of the result.

ing, and resolution of response conflict (Carter et al., 1998; MacDonald, Cohen, Stenger, \& Carter, 2000). More recently, this interest has expanded to more superior regions within the medial frontal lobe, reflecting the recognition that those regions contribute to decision making under uncertainty. Using a natural sampling task in which subjects learned probability rules, Volz and colleagues found that decisions involving outcome uncertainty invoked greater activation in BA 8 than did control decisions without uncertainty (Volz et al., 2003). Furthermore, activation amplitude in this region scaled inversely with probability, providing clear evidence for an uncertainty effect in what they labeled the frontomedian cortex (FMC).

Here, we replicate the basic finding of Volz et al. (2003), in that there was a clear effect of risk upon FMC activation within BA 8/9, but not BA 6 . However, our effect was restricted to the behavioral risk condition, with no differences found for reward risk. The task of Volz and colleagues contained both behavioral and reward risk, the former because their subjects were learning response rules and the latter because rewards were probabilistic. Our simpler task, although less ecologically valid, did not require the subjects to learn stimulus-response contingencies over the course of the experiment, and the subjects were equally fast and accurate in our certainty and reward risk conditions. The results suggest that the FMC activation may reflect subjects' uncertainty as to the needed behavior, and not simply uncertainty in outcome. This difference implies that reward risk, by itself, may not influence processes such as hypothesis testing that have been inferred for this region of the FMC (Elliott \& Dolan, 1998). Note that although the results shown in Figure 5 imply a gradient of activity from posterior to anterior regions of the FMC, it remains possible that there are only two distinct regions: one posteriorly that responds equally to all conditions, and another anteriorly that responds only to behavioral risk. Due to the intrinsic blurring associated with the fMRI hemodynamic response, the anatomical uncertainty following normalization and combining across subjects, and the introduction of a spatial filter, additional targeted studies would be necessary to determine whether a true response gradient exists.

We have recently reported a study of decision making under uncertainty in which subjects made predictions based on the contents of a series of stimuli (Huettel, Song, $\&$ McCarthy, 2005). Although activation was observed in the prefrontal and parietal cortices, as for the present behavioral risk effect, the specific regions differed from those found here. In particular, uncertainty-related activation in the prefrontal cortex was observed in lateral regions (not frontopolar, as here) and in the parietal cortex was observed along the posterior intraparietal sulcus (not the more lateral angular gyrus). On the basis of the regions of activity, we interpreted the fronto-parietal activation as reflecting processes related to cognitive control, in that the task required the subjects to rapidly modify potential response plans as new stimuli were presented (Huettel et al., 2005). This requirement for rapid updating was absent in the present study - and in prior studies of uncertainty (Volz et al., 2003, 2004) - suggesting that the specific manner in which uncertainty is generated determines what brain systems are necessary for its resolution.

A key feature of our experimental design was the use of two separate sets of stimulus-response contingencies in the two experiments. This approach, although it doubled our subject sample, was necessary to remove a potential 


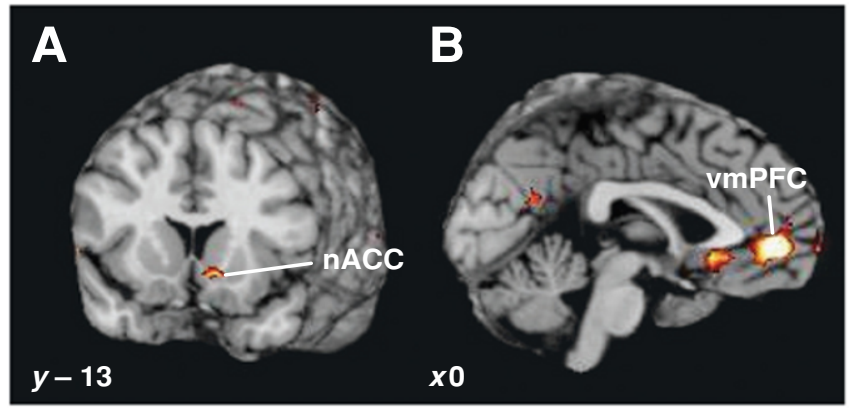

Figure 6. Effects of reward delivery and omission. (A) In the behavioral risk condition, the presentation of a reward signal (in comparison with its omission) evoked significant activity in the nucleus accumbens ( $\mathrm{AACC}$ ). (B) In the reward risk condition, the presentation of a reward signal evoked significant activation in the ventromedial prefrontal cortex (vmPFC).

interpretative confound. If reward magnitudes are held constant while reward probability is manipulated (Experiment 1), expected value will necessarily vary across stimuli. Thus, increased activation in the behavioral risk condition can be attributed to reduced probability of reward or expected value. By keeping expected value constant in Experiment 2 and matching reward probabilities between the reward risk and the behavioral risk conditions, this confound was eliminated. Although our activation maps were generated using data from both experiments, to take advantage of our large sample size, there was good correspondence between the responses observed in each experiment individually. Critically, within all of the regions exhibiting behavioral risk effects (Figures 3-5), similar hemodynamic responses were observed in both experiments. Thus, the observed effects can be attributed to behavioral risk itself, and not to potential covarying factors, such as probability or expected value.

\section{Reward Evaluation}

Stimuli indicating earned rewards, in comparison with those signaling that no reward has been earned, were associated with increased activation in the nucleus accumbens, the ventromedial prefrontal cortex (vmPFC), the lateral orbitofrontal cortex, and several other regions. These regions compose a network involved with evaluating reward information, as has been demonstrated in both single-unit electrophysiology and neuroimaging studies (O'Doherty, 2004). Within this network, there is evidence of functional specialization. The nucleus accumbens, and the ventral striatum more generally, seems to support anticipation of potential rewards on the basis of information about their likelihood and magnitude (Knutson et al., 2001; McClure, Berns, \& Montague, 2003; O’Doherty et al., 2004). The ventromedial prefrontal cortex, however, may support tracking of reward outcomes as they occur (Knutson et al., 2003).

The findings of reward effects in the nucleus accumbens for the behavioral risk condition and in the vmPFC for the reward risk condition are consistent with prior distinctions between these regions. In the latter condition, subjects may treat the stimulus cue as a signal of likely reward, so that activation in the nucleus accumbens, but not in the vmPFC, might transfer to the stimulus cue (Schultz et al., 1997). However, we note that examination of the time courses of activation indicates that both areas evinced reward-related activation in both stimulus types, so any differences are of degree, rather than of kind.

\section{CONCLUSIONS}

In summary, specific regions of the prefrontal, parietal, and insular cortex support decision making when individuals have limited information about optimal behavior. Such behavioral risk can be distinguished from the more typically considered reward risk, in that the former involves uncertainty about needed action, whereas the latter involves uncertainty about potential outcomes. The regions exhibiting behavioral risk effects in the present study were not influenced by reward risk, indicating that they are not sensitive to generalized uncertainty.

The most influential early economic conceptions of risk were predicated on, remarkably enough, introspective assessments of human consciousness (F. H. Knight, 1921). Reasoning that conscious experience reflects imperfect knowledge about the surrounding environment, F. H. Knight presciently described the function of the nervous system as that of anticipating risky future environmental conditions to guide selection of the most adaptive behavior. This perspective has been echoed in modern theories of executive control (R. T. Knight \& Stuss, 2002; Mesulam, 2002; Miller \& Cohen, 2001), emphasizing the potential isomorphisms between behavioral phenomena described by economic theory and underlying cognitive operations.

\section{REFERENCES}

Barraclough, D. J., Conroy, M. L., \& Lee, D. (2004). Prefrontal cortex and decision making in a mixed-strategy game. Nature Neuroscience, 7, 404-410.

Bernoulli, D. (1738). Specimen theoriae novae de mensura sortis. Commentarii Academiae Scientarum Imperialis Petropolitanae, $\mathbf{5}$, 175-192.

Brainard, D. H. (1997). The Psychophysics Toolbox. Spatial Vision, 10, 433-436.

Breiter, H. C., Aharon, I., Kahneman, D., Dale, A., \& Shizgal, P. (2001). Functional imaging of neural responses to expectancy and experience of monetary gains and losses. Neuron, 30, 619-639.

Bunge, S. A. (2004). How we use rules to select actions: A review of evidence from cognitive neuroscience. Cognitive, Affective, \& Behavioral Neuroscience, 4, 564-579.

Carter, C. S., Braver, T. S., Barch, D. M., Botvinick, M. M., Noll, D., \& Cohen, J. D. (1998). Anterior cingulate cortex, error detection, and the online monitoring of performance. Science, 280, 747-749

Clark, V. P., Fannon, S., Lai, S., Benson, R., \& Bauer, L. (2000). Responses to rare visual target and distractor stimuli using event-related fMRI. Journal of Neurophysiology, 83, 3133-3139.

Cohen, J. D., Perlstein, W. M., Braver, T. S., Nystrom, L. E., Noll, D. C., JonidES, J., \& SMith, E. E. (1997). Temporal dynamics of brain activation during a working memory task. Nature, 386, 604-608. 
Courtney, S. M., Ungerleider, L. G., Keil, K., \& Haxby, J. V. (1997). Transient and sustained activity in a distributed neural system for human working memory. Nature, 386, 608-611.

Critchley, H. D., Mathias, C. J., \& Dolan, R. J. (2001). Neural activity in the human brain relating to uncertainty and arousal during anticipation. Neuron, 29, 537-545.

DE FinetTI, B. (1937). La prevision: Ses lois logiques, ses sources subjectives. Annales de l'Institut Henri Poincare, 7, 1-68.

Elliott, R., \& Dolan, R. J. (1998). Activation of different anterior cingulate foci in association with hypothesis testing and response selection. Neurolmage, 8, 17-29.

ElLSBERG, D. (1961). Risk, ambiguity, and the savage axioms. Quarterly Journal of Economics, 75, 643-669.

EPsteIN, L. G., \& WANG, T. (1994). Intertemporal asset pricing under Knightian uncertainty. Econometrica, 62, 283-322.

Fiorillo, C. D., Tobler, P. N., \& Schultz, W. (2003). Discrete coding of reward probability and uncertainty by dopamine neurons. Science, 299, 1898-1902.

FUSTER, J. (1997). The prefrontal cortex: Anatomy, physiology, and neuropsychology of the frontal lobe. Philadelphia: Lippincott-Raven.

Glimcher, P. W. (2003). Decisions, uncertainty, and the brain: The science of neuroeconomics. Cambridge, MA: MIT Press.

Goel, V., \& Dolan, R. J. (2000). Anatomical segregation of component processes in an inductive inference task. Journal of Cognitive Neuroscience, 12, 110-119.

HerrnsteIn, R. J. (1961). Relative and absolute strength of response as a function of frequency of reinforcement. Journal of the Experimental Analysis of Behavior, 4, 267-272.

Huettel, S. A., Mack, P. B., \& McCarthy, G. (2002). Perceiving patterns in random series: Dynamic processing of sequence in prefrontal cortex. Nature Neuroscience, 5, 485-490.

Huettel, S. A., \& McCarthy, G. (2004). What is odd in the oddball task? Prefrontal cortex is activated by dynamic changes in response strategy. Neuropsychologia, 42, 379-386.

Huettel, S. A., Misiurek, J., Jurkowski, A. J., \& McCarthy, G. (2004). Dynamic and strategic aspects of executive processing. Brain Research, 1000, 78-84.

Huettel, S. A., Song, A. W., \& McCarthy, G. (2005). Decisions under uncertainty: Probabilistic context influences activity of prefrontal and parietal cortices. Journal of Neuroscience, 25, 3304-3311.

JiANG, Y., \& KANWISHER, N. (2003a). Common neural substrates for response selection across modalities and mapping paradigms. Journal of Cognitive Neuroscience, 15, 1080-1094.

JiANG, Y., \& KANWISHER, N. (2003b). Common neural mechanisms for response selection and perceptual processing. Journal of Cognitive Neuroscience, 15, 1095-1110.

Johnson, E. J., Hershey, J., Meszaros, J., \& Kunreuther, H. (1993). Framing, probability distortions, and insurance decisions. Journal of Risk \& Uncertainty, 7, 35-51.

Kirino, E., Belger, A., Goldman-Rakic, P., \& McCarthy, G. (2000). Prefrontal activation evoked by infrequent target and novel stimuli in a visual target detection task: An event-related functional magnetic resonance imaging study. Journal of Neuroscience, 20, 6612-6618.

Knight, F. H. (1921). Risk, uncertainty, and profit. Boston: Houghton Mifflin.

Knight, R. T., \& Stuss, D. T. (2002). Prefrontal cortex: The present and the future. In D. T. Stuss \& R. T. Knight (Eds.), Principles of frontal lobe function (pp. 573-597). New York: Oxford University Press.

Knutson, B., Adams, C. M., Fong, G. W., \& Hommer, D. (2001). Anticipation of increasing monetary reward selectively recruits nucleus accumbens. Journal of Neuroscience, 21, 1-5.

Knutson, B., Fong, G. W., Bennett, S. M., Adams, C. M., \& Hommer, D. (2003). A region of mesial prefrontal cortex tracks monetarily rewarding outcomes: Characterization with rapid event-related fMRI. NeuroImage, 18, 263-272.

LockHeAD, G. R. (1992). Psychophysical scaling: Judgments of attributes or objects? Behavioral \& Brain Sciences, 15, 543-601.

MacDonald, A. W., Cohen, J. D., Stenger, V. A., \& Carter, C. S. (2000). Dissociating the role of the dorsolateral prefrontal and anterior cingulate cortex in cognitive control. Science, 288, 1835-1838.
McCarthy, G., Luby, M., Gore, J., \& Goldman-Rakic, P. (1997). Infrequent events transiently activate human prefrontal and parietal cortex as measured by functional MRI. Journal of Neurophysiology, 77, 1630-1634.

McClure, S. M., Berns, G. S., \& Montague, P. R. (2003). Temporal prediction errors in a passive learning task activate human striatum. Neuron, 38, 339-346.

McNeil, B. J., Pauker, S., Sox, H., \& Tversky, A. (1982). On the elicitation of preferences for alternative therapies. New England Journal of Medicine, 306, 216-221.

Mesulam, M. M. (2002). The human frontal lobes: Transcending the default mode through contingent encoding. In D. T. Stuss \& R. T. Knight (Eds.), Principles of frontal lobe function (pp. 8-30). New York: Oxford University Press.

Miller, E. K., \& COHEN, J. D. (2001). An integrative theory of prefrontal cortex function. Annual Review of Neuroscience, 24, 167-202.

O’Doherty, J. P. (2004). Reward representations and reward-related learning in the human brain: Insights from neuroimaging. Current Opinion in Neurobiology, 14, 769-776.

O’Doherty, J. P., Dayan, P., Schultz, J., Deichmann, R., Friston, K., \& Dolan, R. J. (2004). Dissociable roles of ventral and dorsal striatum in instrumental conditioning. Science, 304, 452-454.

Paulus, M. P., Hozack, N., Frank, L., \& Brown, G. G. (2002). Error rate and outcome predictability affect neural activation in prefrontal cortex and anterior cingulate during decision-making. NeuroImage, 15, 836-846.

Paulus, M. P., Hozack, N., Zauscher, B., McDowell, J. E., Frank, L., Brown, G. G., \& Braff, D. L. (2001). Prefrontal, parietal, and temporal cortex networks underlie decision-making in the presence of uncertainty. NeuroImage, 13, 91-100.

Pelli, D. G. (1997). The VideoToolbox software for visual psychophysics: Transforming numbers into movies. Spatial Vision, 10, 437-442.

Pochon, J. B., Levy, R., Fossati, P., Lehericy, S., Poline, J. B., PILlon, B., ET AL. (2002). The neural system that bridges reward and cognition in humans: An fMRI study. Proceedings of the National Academy of Sciences, 99, 5669-5674.

RAMSEY, F. P. (1931). The foundations of mathematics and other logical essays. New York: Harcourt, Brace.

SavaGe, L. J. (1954). Foundations of statistics. New York: Wiley.

Schultz, W. (1998). Predictive reward signal of dopamine neurons. Journal of Neurophysiology, 80, 1-27.

Schultz, W., Dayan, P., \& Montague, P. R. (1997). A neural substrate of prediction and reward. Science, 275, 1593-1599.

Schumacher, E. H., Elston, P. A., \& D'Esposito, M. (2003). Neural evidence for representation-specific response selection. Journal of Cognitive Neuroscience, 15, 1111-1121.

SIMON, J. R., \& BAKER, K. L. (1995). Effect of irrelevant information on the time to enter and retrieve relevant information in a Stroop-type task. Journal of Experimental Psychology: Human Perception \& Performance, 21, 1028-1043.

Simon, J. R., \& SMALl, A. M. (1969). Processing auditory information: Interference from an irrelevant cue. Journal of Applied Psychology, 53, 433-435.

Stevens, A. A., Skudlarski, P., Gatenby, J. C., \& Gore, J. C. (2000). Event-related fMRI of auditory and visual oddball tasks. Magnetic Resonance Imaging, 18, 495-502.

Ullsperger, M., \& VON Cramon, D. Y. (2001). Subprocesses of performance monitoring: A dissociation of error processing and response competition revealed by event-related fMRI and ERPs. NeuroImage, 14, 1387-1401.

Volz, K. G., Schubotz, R. I., \& von Cramon, D. Y. (2003). Predicting events of varying probability: Uncertainty investigated by fMRI. NeuroImage, 19, 271-280.

Volz, K. G., Schubotz, R. I., \& von Cramon, D. Y. (2004). Why am I unsure? Internal and external attributions of uncertainty dissociated by fMRI. NeuroImage, 21, 848-857.

(Manuscript received January 24, 2005; revision accepted for publication October 17, 2005.) 